
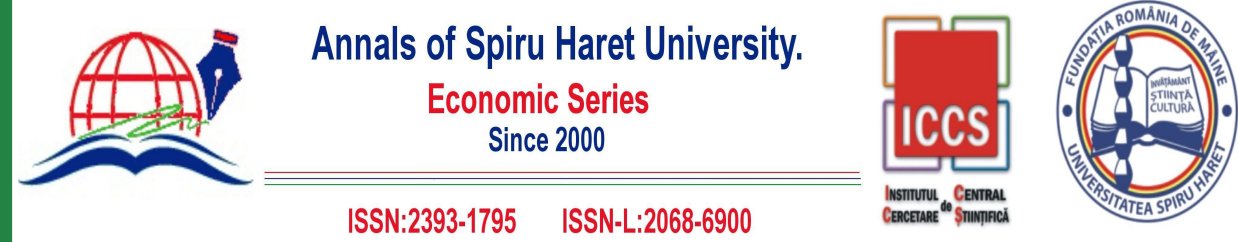

Issue 2/2019

\title{
IMPACT OF ARTIFICIAL INTELLIGENCE ON AGRICULTURAL, HEALTHCARE AND LOGISTICS INDUSTRIES
}

\author{
Aydan ABDULLAYEVA ${ }^{1}$ \\ ${ }^{1}$ Bachelor in Marketing, Azerbaijan State Oil and Industry University, \\ 16/21 Azadliq Ave, Baku, Azerbaidjan, Tel: +994124934557, \\ Email: aydan.abdullayeva.99@mail.ru
}

How to cite: ABDULLAYEVA, A. (2019). "Impact of Artificial Intelligence on Agricultural, Healthcare and Logistics Industries." Annals of Spiru Haret University. Economic Series, 19(2), 167-175, doi: https://doi.org/10.26458/1929

\begin{abstract}
This qualitative research study was conducted to illustrate the relationships between Artificial Intelligence (AI) and non-tech businesses. AI is a broad branch of computer science. In information technology, the intelligent machine is a compliant and logical agent that recognizes its environment and takes full advantage of opportunities to achieve something. This paper provides detailed examples using AI outside of IT. The main method which is used for this research is literary analysis. The article consists of explanations about artificial intelligence in general, its impacts on logistics and transportation, agriculture and healthcare industries. Moreover, in this article, the methods used to leverage the developments of aforementioned industries are also mentioned and
\end{abstract} discussed.

Keywords: artificial intelligence; machine learning; information technology; agriculture; healthcare; transportation.

JEL Classification: $\mathrm{O} 32$ 


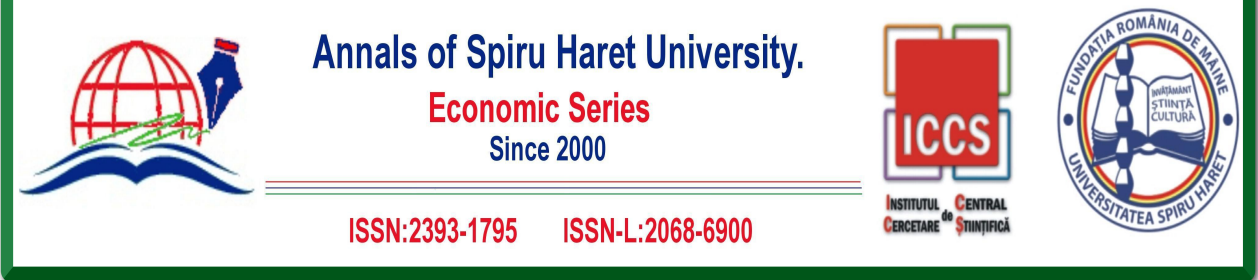

Issue 2/2019

\section{Introduction}

Nowadays, more and more companies and organizations opt for using Artificial Intelligence for various purposes because of the fact that AI is now a really powerful tool that can help those companies stand out in the competition with their rivals, ease their tasks, and surely reduce the chance of making errors. AI tools signal the beginning of a new era with the help of making critical decisions. However, it doesn't generate forecasts. While there might be some problems being caused by using AI for analyzing customers or letting it decide instead of humans, it still helps a lot and allow firms save time and use it in a better way. Of course, almost all companies inside the IT world in 21st century use AI because now artificial intelligence has captured every area of the technology in developed regions of the world. However, we can also easily observe that companies outside of IT industry make efforts to use AI tools too.

\section{Research Methodology}

The present study was analyzed based on the primary and secondary data. Qualitative research method was used for comprehensive explanations about impacts of AI on industries which are not directly related to information technology. TED video recordings were examined as a primary data. "How can AI influence sectors other than IT?" - to find out the possible answers to this question, secondary data tools were also applied. From the other types of qualitative data collection tools I used 2 types of participatory research methods - industry mapping and community meeting. This community meeting was held on December 21-22. It was a seminar called "What is AI and its role in healthcare?" Industry mapping helped this research to provide better understanding about potential effects of machine learning on different sectors. The mostly used method in this paper was literature analysis.

\section{Understanding Artificial Intelligence (AI)}

Artificial intelligence (AI), sometimes called machine intelligence, is intelligence demonstrated by machines, in contrast to the natural intelligence displayed by humans and other animals. In other words, it is an area of computer science that emphasizes the creation of intelligent machines that work and react like humans. Until recently, computers could do only what we already programmed them to do. But now, they can learn it themselves and can show us the result that we have never 


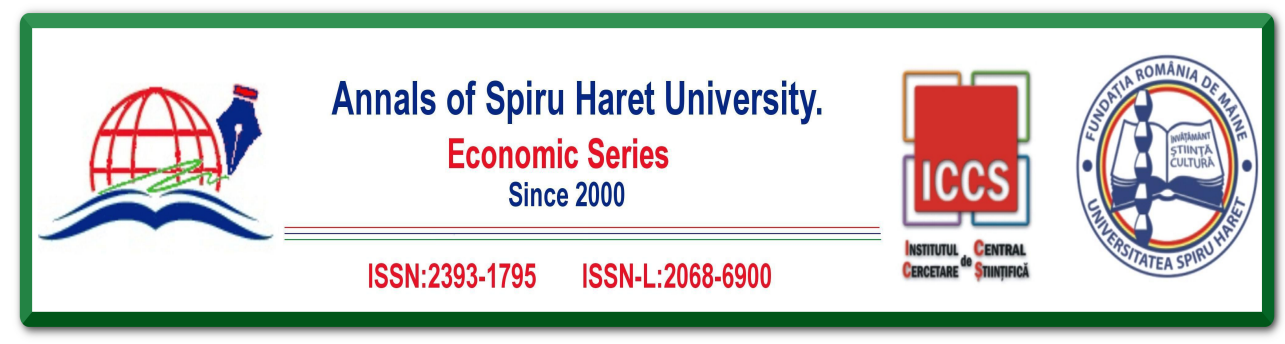

Issue 2/2019

told them before. It's true that press can sometimes easily exaggerate the AI by replacing it with something like Terminator or something much smarter than humans that we can currently see only in sci-fi movies. Experts refer to this specific instance of AI as artificial general intelligence, and if we do ever create something like this, it'll likely be a long way in the future. In reality, AI is used for various purposes including learning (the acquisition of information and rules for using the information), reasoning (using rules to reach approximate or definite conclusions) and self-correction. Particular applications of AI include expert systems, speech recognition and machine vision. Actually, now it affects every aspect of our life, whether we like it, or not. It's being deployed in health care and warfare; it's helping people make music and books; it's scrutinizing your resume, judging your creditworthiness, and tweaking the photos you take on your phone. When trying to understand AI, it is better to talk about machine learning rather than AI. The machine can reach a new, accurate conclusion based on what it has learned in the past. The crucial thing to understand about $\mathrm{AI}$ is that the machine is not programmed with all the possible answers but uses what it has learned to create new answers. This is a subfield of artificial intelligence that encompasses almost all the methods having the biggest influence on the world right now. So, how does Machine Learning work?

The point experts in the area are making is that we're now at the point where AI is going to get normal fast. "Eventually, pretty much everything will have machine learning somewhere inside and no-one will care," says Benedict Evans, a venture capital strategist. Kai-Fu Lee, another venture capitalist and former AI researcher, describes the current moment as the "Age of implementation" - one where the technology starts "spilling out of the lab and into the world".

It's obvious that when we say "into the world", we mainly mean businesses.

There are a lot of ways in each ever growing business sector that AI can be used to achieve ultimate goals of particular organizations. According to the research, while $15 \%$ of organizations used machine intelligence in 2018 , this number will be $31 \%$ by the end of 2019. Another research shows AI-focused start-ups have seen a 14-fold increase since 2000. That being said, consumers in most cases don't know when they are interacting with AI, as just over $30 \%$ of them said they use an AI-powered service or device, when the number who actually does is 77\%. [Uthermohlen, 2018] These numbers given above is enough to characterize the importance of artificial intelligence in business world and in this article I will make efforts to describe AIusage in non-tech parts of business markets and provide examples where relevant. 


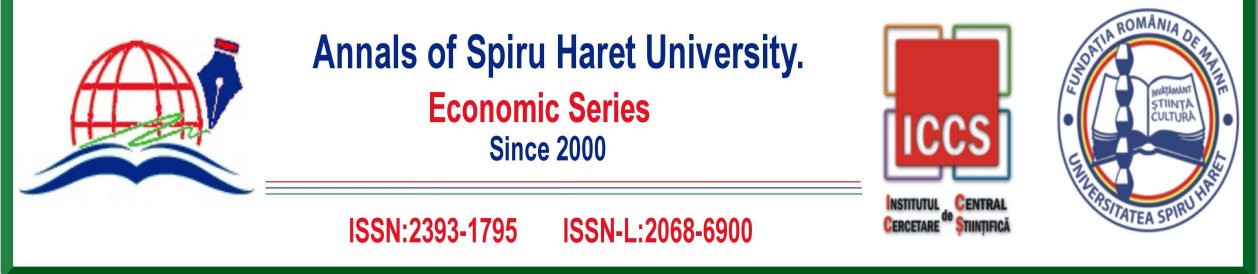

Issue 2/2019

\section{AI in Logistics, Supply Chain and Transportation}

A myriad of professionals in this sector believe that logistics, supply chain and transportation are among the fastest-changing industries in all over the world. But of the most powerful forces that affect the development of this industry, AI and machine learning are always at the top. Leading organizations are already using brand-new technologies to work with core strategies and to solve the problems related to carriers, inventories, staff, vehicles, and so on.

While we know that application of technologies of this kind to the industry brings a lot of data, it is also a fact that logistics and transportation industry is getting data for many years. Therefore, the main difference between today's and yesterday's transportation industry is not only the amount of data being captured but also more advanced computer power and algorithms available to classify, examine and quicken understanding and operation. The industry which has always been data-focused, today can utilize artificial intelligence, machine learning, and data-mining for not only overall strategies and planning but also for real-time decision-making processes.

Let's look at some ways that AI and associated technologies are used to revolutionize logistics, supply chain and transportation industry.

\section{Predictive Analysis}

As in each sector, machine intelligence is used to foresee, for example, transportation needs to avoid breakdowns and thus reducing the chance of failing to meet customer expectations.

\section{Strategic Optimization}

Computers using artificial intelligence can gather, analyze information to make effective decisions within the matter of seconds and save time for humans.

\section{Robotics}

Robots are already being used widely for instance, inside warehouses to move, track and locate inventories. Consequently, it's not a coincidence that the sales of logistics and warehousing robots globally will reach $\$ 22.4$ billion by 2022 . [Tractica, 2018]

\section{Autonomous vehicles}

Last but not the least, the usage of driverless cars are inevitable and maybe the organizations who can take advantage of it the most are companies in this sector because using self-driving vehicles they can save plenty of money which is paid as the wages of drivers. 

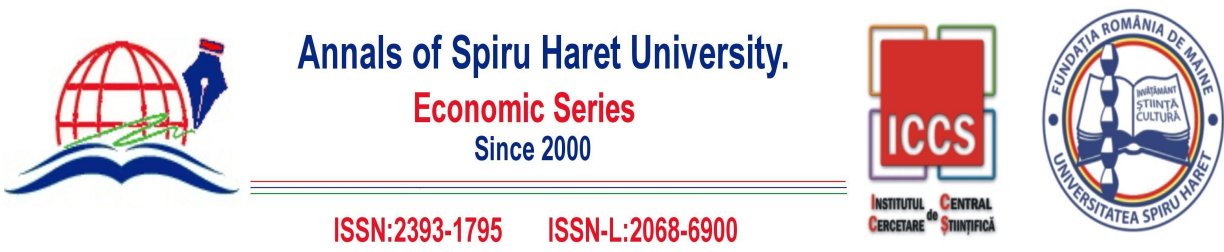

Issue 2/2019

\section{AI in Agriculture}

Agriculture is among the fields where AI is used and potentially can be used to achieve great results in both productivity and effectiveness. Taking into account the fact that agriculture is one of the major parts of a lot of countries' economy (including some developing and developed countries), we can easily comprehend how important the widespread use of artificial intelligence and machine learning algorithms would be for the global economic situation.

There are some necessary factors such as climate change, the demographic problems and reasonable concerns in food security that encourage people and organizations in this industry to seek more innovative ways to protect and improve crop lands. And AI is nowadays becoming the number one priority for those trying to take part in the technological evolution of the industry.

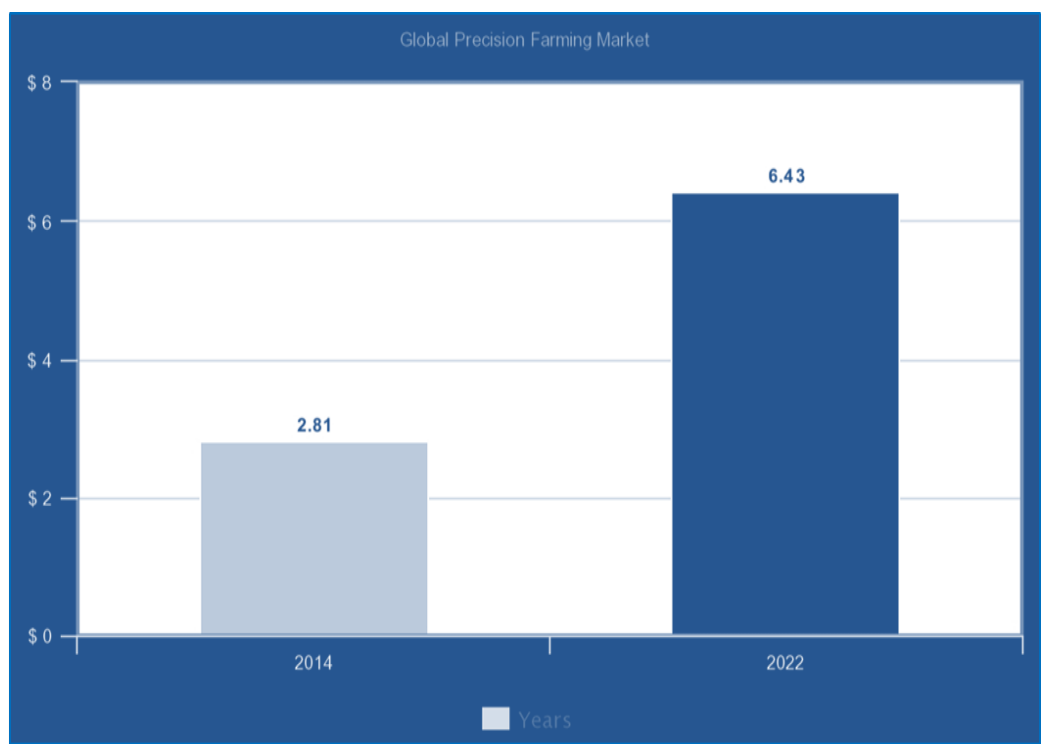

Fig. 1. Global Precision Farming Market

To start with, it must be stated that, North America and Europe are the biggest and the most developed markets to benefit from advanced technologies in the agricultural 


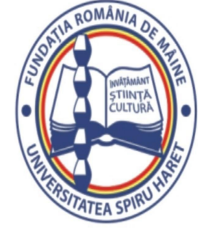

Issue 2/2019

field. However, it is also notable that Asia, among all other continents, including N. America and Europe, has the greatest and excellent potential for growth.

As mentioned above, technology is becoming an inseparable tool of every agronomist and farmer in today's agricultural industry. As a result, being born in 1990, the phrase of "Precision Agriculture" is turning out to be more popular every other year. In short, Precision Agriculture is the usage of technological advancements such as GPS guidance, robotics, control systems, drones, autonomous vehicles, sensors, etc. in an effort to make farming more accurate and controllable. According to Statistics MRC, the Global Precision Farming market accounted for \$2.81 billion in 2014 and is expected to grow at a CAGR of $12.5 \%$ to reach $\$ 6.43$ billion by 2022 . Approximately $70-80 \%$ of all new equipment purchases in the field contain some sort of precision agriculture tools and by the end of 2020s the vast majority of farmers, if not all, will use precision farming in all over the world.

When it comes to the application of AI and machine learning to this industry, it falls into three main classifications.

\section{Agricultural Robots}

Companies are developing self-controlling robots for everyday agricultural tasks like harvesting, planting, seeding and so on, which complete their tasks at a faster pace than human labourers.

For example, a company "Blue River Technologies" developed a robot named "See\&Spray" to monitor and spray weeds on cotton plants.

\section{Crop and Soil Monitoring}

Undoubtedly, checking and maintaining the soil and crop health are one of the most essential agricultural operations. In order to replace humans and thus, avoiding the risk of failures, Berlin-based start-up - "PEAT" developed an application called "Plantix" which can examine the health status of soil.

\section{Predictive Analytics}

Machine learning algorithms can be used to predict various environmental impacts on crop yield. "aWhere" - a company located in Colorado - uses machine learning along with satellites to predict weather and to check whether the crop has disease(s) or not. 


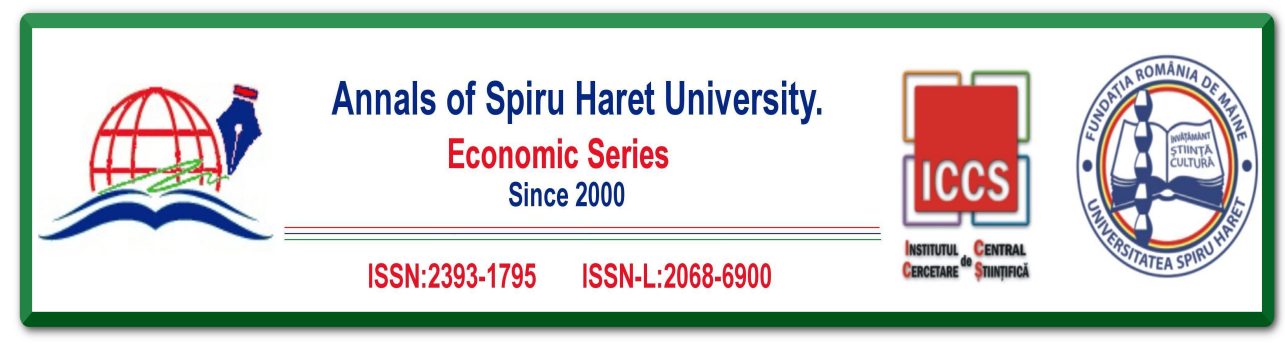

Issue 2/2019

\section{AI in Healthcare}

It's not a secret to anyone that artificial intelligence is hugely impacting the development of healthcare, regardless of its branches. Doctors are already using AI and related technologies, however, mainly in developed regions of the world. But taking the current developing speed into account, it can be easily predicted that in a short amount of time AI will help doctors in all over the world. In healthcare, AI is changing the game with various applications and decision support image analysis. Decision support systems quickly analyze large amounts of data within the electronic medical record. AI technology is also taking the uncertainty out of viewing patient scans by highlighting problem areas on images aiding in the screening and diagnosis process. Artificial intelligence helps with the issue of physician burnout by collecting patient data via an app or text messaging. AI integration working enables smarter solutions to a variety of issues for patients, hospitals and the healthcare industry.

According to the research carried out by "Accenture", AI healthcare space is estimated to reach $\$ 6.6$ billion by 2021 .

It would be possible to outline 8 ways that AI is transforming the healthcare industry like never before, of which we present the followings:

\section{The AI diagnosis}

The research found out that up to 440,000 people die every year in the USA because of preventable medical errors. [PSA, 2014] As we know, the main reason behind it is diagnosis. But today, AI is playing a major role in the evolution of medical diagnosis. For example, chat bots now ask patients a series of question regarding their symptoms taking the guesswork out of self-diagnosis and saving both the patient and provider time and money. Deep learning algorithms are also used in oncology, recognizing cancerous tissues just like trained physicians. Furthermore, facial recognition software can be combined with machine learning algorithms to help clinicians detect rare genetic diseases.

\section{Robot-assisted surgery}

Robotic surgery allows doctors to operate many kinds of procedures without any errors and with more flexibility and control in comparison to traditional techniques. Despite still existing small risks, robot-assisted surgery has lots of benefits including fewer surgical site infections, less blood loss, smaller scars, and of course, quicker recovery. 


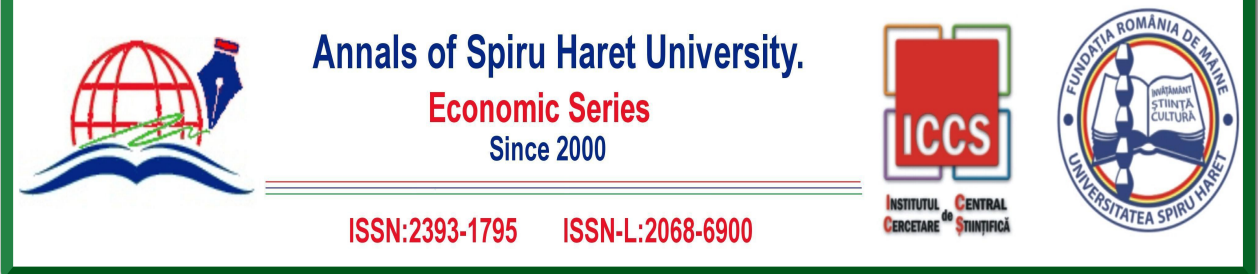

Issue 2/2019

\section{AI in drug development}

Machine intelligence is no longer a science-fiction for drug discovery. In fact, computational power has been used for this purpose for nearly 40 years. But the real difference with $\mathrm{AI}$ is that it can learn itself and can tell us which molecules will be successful for certain application

\section{Conclusion}

To recapitulate, it has to be stated that artificial intelligence is now quite a dynamic and influential thing which is being improved at a faster pace than expected. And keeping it in mind, virtually every business which can afford it tries to use it for different objectives including increasing productivity and effectiveness, saving time, money and etc. While in logistics, supply chain and transportation and agricultural industry, the main use of this technology is majorly easing the everyday tasks, in medical industry, though its application to this industry is much more complicated than those, we talk about saving human lives and providing better healthcare for the patients.

\section{References}

[1] Breazeal, C. L. (2002). Designing sociable robots. MIT press.

[2] Coates, J. F. (1995). Agriculture in the 21st century. Technological Forecasting and Social Change, 50(1), 105-109.

[3] Hoffman, G., \& Breazeal, C. (2004, September). Collaboration in human-robot teams. In AIAA 1st Intelligent Systems Technical Conference (p. 6434).

[4] Jiang, F., Jiang, Y., Zhi, H., Dong, Y., Li, H., Ma, S., ... \& Wang, Y. (2017). Artificial intelligence in healthcare: past, present and future. Stroke and vascular neurology, 2(4), 230-243..

[5] King, A. (2017). The future of agriculture. Nature, 544(7651), S21-S23.

[6] Lintonen, T. P., Konu, A. I., \& Seedhouse, D. (2007). Information technology in health promotion. Health Education Research, 23(3), 560-566.

[7] Min, H. (2010). Artificial intelligence in supply chain management: theory and applications. International Journal of Logistics: Research and Applications, 13(1), 13-39.

[8] Mitul Makadia.(2018). Everything you need to know about AI in healthcare. https://lesgtyconge.ml/everything-you-need-to-know-about-ai-in-healthcare

[9] Neill, D. B. (2013). Using artificial intelligence to improve hospital inpatient care. IEEE Intelligent Systems, 28(2), 92-95. 

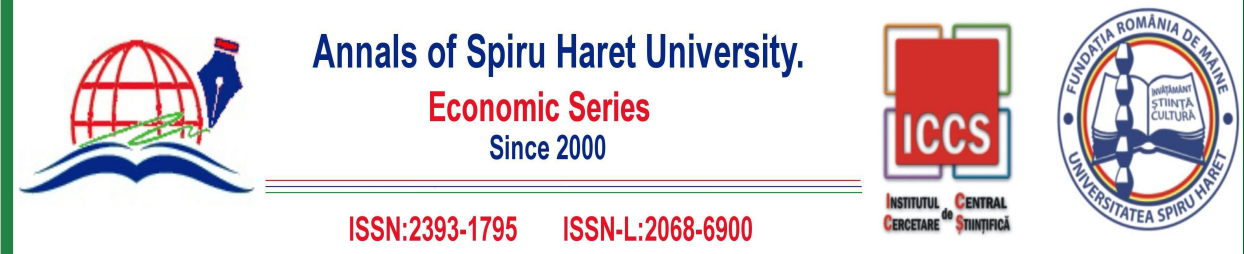

Issue 2/2019

[10] Ramesh, A. N., Kambhampati, C., Monson, J. R., \& Drew, P. J. (2004). Artificial intelligence in medicine. Annals of The Royal College of Surgeons of England, 86(5), 334.

[11] Reddy, S. (2018). Use of Artificial Intelligence in Healthcare Delivery. In eHealthMaking Health Care Smarter. IntechOpen.

[12] Robinson Adam (2019). The top 5 changes that occur with AI in logistics, https://cerasis.com/ai-in-logistics/.

[13] Stafford, J. V. (2000). Implementing precision agriculture in the 21 st century. Journal of Agricultural Engineering Research, 76(3), 267-275.

[14] Training, C. D., \& Board, M. H. S. The Red (Team) Analysis Society.

[15] Wang, F. Y. (2008). Toward a revolution in transportation operations: AI for complex systems. IEEE Intelligent Systems, 23(6), 8-13.

[16] https://www.forbes.com/sites/insights-penske/2018/09/04/how-artificial-intelligenceand-machine-learning-are-revolutionizing-logistics-supply-chain-andtransportation/\#49f4cb0f58f5

[17] https://www.supplychain247.com/article/6_ways_artificial_intelligence_technology_i s_impacting_the_supply_chain

[18] https://www.technologynetworks.com/informatics/articles/artificial-intelligence-realsolutions-how-ai-can-accelerate-drug-discovery-311063

[19] https://agfundernews.com/what-is-precision-agriculture.html

[20] https://thenextweb.com/contributors/2018/12/22/3-ways-ai-will-improve-healthcarein-2019/

[21] https://www.forbes.com/sites/bernardmarr/2018/07/27/how-is-ai-used-in-healthcare-5powerful-real-world-examples-that-show-the-latest-advances/\#fd98cb85dfbe

[22] https://novatiosolutions.com/10-common-applications-artificial-intelligencehealthcare/ 
